\title{
A Climate Event Portal for Citizen Science
}

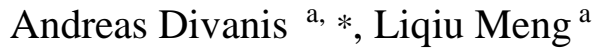 \\ ${ }^{a}$ Chair of Cartography, Technical University of Munich, 80333 Munich, Germany, andreas.divanis@tum.de, liqiu.meng@tum.de \\ * Corresponding author
}

Keywords: Citizen Science, Open Data Portal, Mobile, BAYKLIF, Climate Event Portal, Climate Change

\begin{abstract}
:
Climate change as both a local and global phenomenon causes numerous societal and financial implications every year. Its perception among citizens, however, is highly controversial. For example, in August 2018, the negative effects of climate change were very prominent in the state of Bavaria, when farmers lost large amounts of feed crop due to a drought in the same year. While governments can initiate policies in response to this, implementing these policies in a democracy requires the approval of the public, which is mainly composed of non-experts. In order to mobilize citizens for their own benefit, thus promote citizen science, the Bavarian Climate Research Network (BAYKLIF) was launched. One of the objectives deals with the creation of a portal and a number of mobile services for citizens, domain specialists and decision makers. This paper is dedicated to a project within this network, which is relevant to cartography. It aims to create a Climate Event Portal accessible by normal citizens who may also contribute with their own data to the portal or validate existing climate events based on their knowledge and supported by visualization tools. The anticipated portal consists of five core concepts.
\end{abstract}

First, the OpenEventCollector, which will search for climate events by categories and collect event data in an interactive and intuitive way. Second, an OpenEventCrawler, that should automatically search open digital archives and social media, thus keep generating data about climate events. Third, an OpenEventEditor, to visualize the data for the user, allow them to interact with the data, and contribute their own (Fig. 1). This, however, must all be possible within the technical limitations of smartphones or web-applications. Fourth, an OpenEventDetector, which should automatically detect climate events from datasets, via machine learning. Fifth, an OpenEventAnalysor that explains the context of climate events, the visualizations and the process of generating this data.

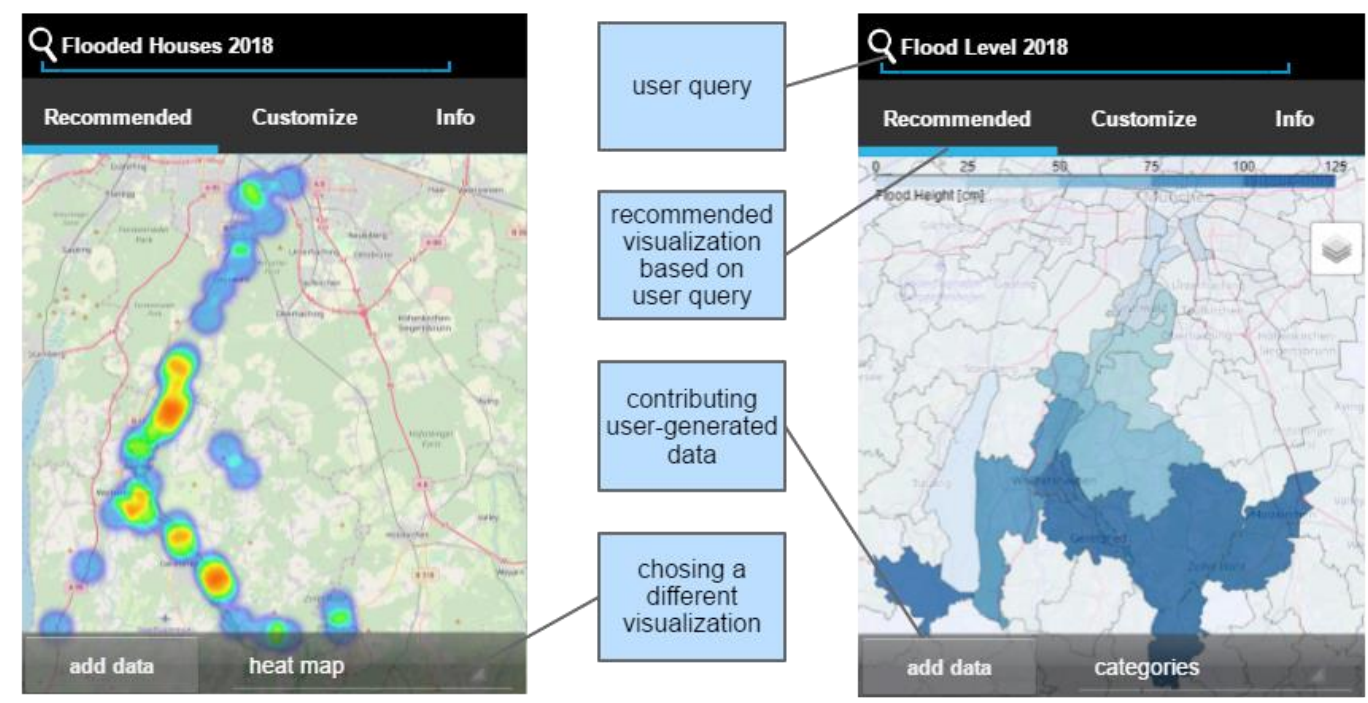

Figure 1. Mock-up on climate-event-portal - user interaction

The purpose of this Climate Event Portal is to involve the user, thus ensuring that all data permits the re-use is necessary. Its development reveals three interrelated challenges: (1) examining user-generated data for relevance and legal compliance, (2) improving representative user feedback, which requires the participation of diverse user groups and (3) sustaining users' interest and attention, which requires innovative incentives for users' contributions (e.g. gamificationoriented visualization, visual storytelling etc.). This work will contribute to the knowledge on how to adapt presentations based on citizens' interaction, as well as create an event portal, where citizen-contributed data and expert-generated data are coalesced. The authors aim to share their experiences and findings with researchers engaged in similar projects of citizen science. 